\title{
MELODÍAS PARA CONFIRMAR, PREGUNTAR, SUGERIR O PEDIR EN ESPAÑOL
}

\author{
Dolors Font-Rotchés \\ Universidad de Barcelona \\ dolorsfont@ub.edu
}

Miguel Mateo-Ruiz

Universidade Federal de Rio de Janeiro

miquel.mateo@gmail.com

\begin{abstract}
Resumen
En este artículo, vamos a describir cuatro melodías /+interrogativas/ del español, obtenidas con el método Análisis Melódico del Habla (AMH), a partir de un corpus procedente de todo el territorio peninsular y Canarias, con la finalidad de que puedan ser utilizadas en el aprendizaje de esta lengua. Se han analizado un total de 282 enunciados-pregunta, producidos por 197 informantes, entre hombres y mujeres de edades distintas, y en los que la respuesta esperada es sí/no. De cada melodía tipo, se describen sus rasgos melódicos, se ejemplifica y se presentan sus usos pragmáticos más relevantes hallados en el corpus. Se constata que estos enunciados no se utilizan exclusivamente para preguntar y obtener una respuesta más o menos conocida, sino que con frecuencia conllevan otras acciones intencionales, como invitar, confirmar, sugerir, pedir permiso, ofrecer, expresar cortesía o, simplemente, se trata de una pregunta retórica de la que no se espera respuesta.
\end{abstract}

Palabras clave: entonación, usos pragmáticos, melodías interrogativas, español

\section{Resum}

En aquest article, descriurem quatre melodies /+interrogatives/ de l'espanyol, les quals han estat obtingudes amb el mètode Anàlisi Melòdica de la Parla (AMP), a partir d'un corpus procedent de tot el territori peninsular i de Canàries, amb la finalitat que es puguin utilitzar en l'aprenentatge de llengües. S'han analitzat un total de 282 enunciats-pregunta, produïts per 197 informants, entre homes i dones d'edats diverses, dels quals s'espera una resposta de sí/no. De cada melodia tipus, es descriuen els seus trets melòdics, s'exemplifica i es detallen els seus usos pragmàtics més rellevants trobats en el corpus. Es constata que aquests enunciats no solament es fan servir per preguntar i obtenir una resposta més o menys coneguda, sinó que molt sovint comporten altres accions intencionals, com convidar, confirmar, suggerir, demanar permís, oferir, expressar cortesia, o, simplement, es tracta d'una pregunta retòrica de la qual no s'espera resposta.

Paraules clau: entonació, usos pragmàtics, melodies interrogatives, espanyol 


\begin{abstract}
In this paper, we describe four melodies /+interrogatives/ of Spanish which have been obtained by applying the Melodic Analysis of Speech (MAS) method to a corpus coming from the whole peninsular territory and the Canary Islands, in order to use them in language learning. We have analysed a total of 282 question settings by 197 speakers, among men and women of different age, of which the expected answer is yes or no. We will describe the melodic features of each melody type; exemplify each one and detail the more relevant pragmatic uses found in the corpus. We state that these utterances are used not only to ask for and obtain a more or less known answer but also they often involve other intentional actions such as inviting, confirming, suggesting, offering, expressing politeness 0 , simply, it is a rhetorical question of which we do not expect any answer.
\end{abstract}

Keywords: intonation, pragmatic uses, interrogative melodies, Spanish

\title{
1. INTRODUCCIÓN
}

En este artículo, vamos a describir las melodías /+interrogativas/ del español, obtenidas con el método Análisis Melódico del Habla $(A M H)$ a partir de un corpus amplio de hablantes de todo el territorio peninsular y Canarias, y su validación en pruebas perceptivas ${ }^{1}$. De cada una, aportaremos sus rasgos melódicos y fonológicos, las ejemplificaremos con enunciados producidos por informantes anónimos en habla espontánea y presentaremos sus usos pragmáticos más relevantes hallados en el corpus (confirmar, preguntar, sugerir, realizar una petición o un ofrecimiento, pedir permiso, entre otros) con la finalidad de que estas melodías puedan ser utilizadas en el aprendizaje del español. Para esta clasificación, nos hemos basado en la propuesta de tipología de preguntas que Escandell-Vidal (1998a, 1998b, 2002) establece a partir de la teoría de la relevancia de Sperber y Wilson (1986) y Wilson y Sperber (1993).

Desde los estudios pioneros de Navarro Tomás (1944) hasta nuestros días, diversos trabajos desde presupuestos teóricos distintos han abordado la entonación de las interrogativas absolutas. En general, han descrito la curva melódica de este tipo de enunciados mediante un tono descendente en el cuerpo del grupo fónico y una inflexión final ascendente. En este sentido, encontramos estudios que siguen el modelo métrico-autosegmental (Face, 2004; Estebas y Prieto, 2010; Vizcaíno y Cabrera, 2011) o las propuestas del proyecto Amper en el dominio hispánico (Martínez Celdrán y Fernández Planas, 2003; Elejabeitia et al., 2005; Ramírez, 2005; Dorta, 2007; Martínez Celdrán, 2011; Congosto, 2011), entre otros. Cabe decir que trabajos como los de Quilis (1989), Dorta y Hernández (2005), y Vizcaíno y Cabrera (2011) reconocen además la existencia de otro patrón con inflexión final circunfleja ascendente-descendente en Las Palmas de Gran Canaria. Todos ellos basan sus estudios en habla de laboratorio con un número muy limitado de informantes, que han sido inducidos, con estrategias diversas, a formular el tipo de pregunta que se necesitaba para realizar el estudio, o bien, se les ha hecho leer unas frases preparadas ad hoc.

También, desde el método Análisis Melódico del Habla (AMH), propuesto en Cantero (2002) y que se centra en el análisis y descripción de la entonación del habla espontánea, se han

\footnotetext{
${ }^{1}$ La investigación que presentamos se enmarca dentro de los trabajos del Grup de Recerca Entonació i Parla (consolidado por la Generalitat de Catalunya, desde 2009) y del proyecto Análisis Habla y Modelos Didácticos (FFI2013-41915-P), subvencionado por el Ministerio de Economía y Competitividad.
} 
descrito las interrogativas absolutas (Cantero et al., 2002, y Cantero y Font-Rotchés, 2007). Los autores, partiendo de un corpus de televisión genérico de seis horas en el que no se hacía distinción entre las diversas zonas dialectales de la península y con diversos informantes anónimos, aportaron nuevos patrones interrogativos, descritos con rasgos melódicos concretos y objetivos (dos neutros con inflexión final ascendente, II y III, y uno enfático con inflexión final circunfleja ascendente-descendente, IV). Font-Rotchés y Mateo (2011), basándose en un corpus mucho más amplio (más de cien horas de material audiovisual), han constatado la existencia de un nuevo patrón de cuerpo e inflexión final ascendentes, el XIII, $\mathrm{y}$, posteriormente, han tratado el rendimiento y el uso pragmático de estos cuatro patrones en el sur de la península (Andalucía, Extremadura, Murcia, Castilla-La Mancha) y Canarias (Font-Rotchés y Mateo, 2013).

Aunque el estudio de la entonación de las interrogativas absolutas ha interesado a los investigadores, especialmente en la última década, los resultados que se han obtenido aún no se reflejan en los manuales para aprender español como lengua extranjera ampliamente usados, como podrían ser, Gente 1 y 2 (Sans y Martín Peris, 2004), Vuela 1, (Alvárez et al., 2005) o El ventilador (Chamorro et al., 2006), como evidencian Baditzné (2012) y Baditzné y Font-Rotchés (2013). Las autoras constatan que en estos manuales no existe ningún apartado ni actividad que trate el fenómeno de la entonación de forma directa. Solo aparecen modelos de entonación en diálogos leídos por locutores en los audios que aportan, los cuales tienen relación con las actividades orales propuestas a los aprendientes. Además de tratar la entonación de forma indirecta, las preguntas que aparecen en estos diálogos se caracterizan por pertenecer casi todas a un patrón ascendente (II o III) y solo, en casos muy aislados, a un patrón enfático (IV, con inflexión final ascendente-descendente, o XIII, con cuerpo e inflexión final ascendentes).

En la Nueva gramática de la lengua española. Fonética y fonología (RAE, 2011) tampoco se considera toda la variedad entonativa de este tipo de enunciados. Se describe un patrón neutro ascendente general y uno descendente en Canarias, Asturias, Galicia y Cataluña. En cuanto a las preguntas no neutras, además de un patrón con final ascendente y un campo tonal más o menos amplio, se describe otro circunflejo para las relativas y las exhortativas.

Por consiguiente, la finalidad de este trabajo es presentar los modelos de la entonación interrogativa, obtenidos después de analizar 282 enunciados y de su validación en pruebas perceptivas, junto con la descripción de sus usos pragmáticos en los distintos contextos para que puedan ser utilizados en los niveles sucesivos de aprendizaje del español y puedan beneficiar a los aprendientes en la adquisición de una buena competencia lingüística.

\section{EL CORPUS Y EL MÉTODO ANÁLISIS MELÓDICO DEL HABLA (AMH)}

Los contornos analizados en este estudio provienen principalmente de un corpus de enunciados de distintas zonas de la península y de Canarias (Ballesteros, 2011, y Mateo, 2014). El número total de enunciados supera los 2400 y fueron obtenidos de más de 650 informantes a partir de unas 100 horas de grabaciones de programas televisivos ${ }^{2}$. De este corpus, se han seleccionado 236 enunciados interrogativos que, en el contexto de su emisión, correspondían a preguntas en las que la respuesta esperada era sí/no. Cabe decir que también

\footnotetext{
${ }^{2}$ Para una descripción detallada sobre el procedimiento de selección y obtención del corpus, véase el trabajo de Ballesteros et al. (2010).
} 
hemos considerado aquellos enunciados interrogativos cuya fuerza ilocutiva puede ser diferente, es decir, conlleva otras acciones intencionales (una petición, una confirmación, un reto, una sugerencia, un ofrecimiento, entre otros) o, simplemente, se trata de una pregunta retórica, de la que no se espera respuesta. Y se completa con 46 enunciados de composición aleatoria: 21 del corpus de español elaborado previamente en el Laboratorio de Fonética Aplicada de la Universitat de Barcelona (Cantero et al., 2002) y 25 procedentes de la tesis doctoral de Baditzné (2012).

Todos ellos se han obtenido en programas de televisión de canales generales y autonómicos españoles y, en el caso de Baditzné, proceden de Youtube. Fueron emitidos en contextos de debates, concursos, entrevistas, reportajes temáticos y programas similares.

Se han analizado, pues, un total de 282 enunciados, producidos por 197 informantes -115 mujeres y 82 hombres- hablantes nativos de castellano con edades comprendidas entre los 16 y los 83 años en el momento de la emisión de los enunciados y de composición totalmente aleatoria por lo que respecta tanto a la ubicación dialectal concreta como al origen social y nivel cultural.

La metodología utilizada es de base empírica y experimental y se centra en los presupuestos de la teoría del Análisis Melódico del Habla (AMH), expuestos de forma detallada en Cantero (2002) y Font-Rotchés (2007), y el protocolo de análisis utilizado se describe en Cantero y Font-Rotchés (2009) ${ }^{3}$.

Se trata de un método muy adecuado para la investigación y descripción detallada de la entonación de las lenguas y cuyos resultados son útiles para desarrollar aplicaciones didácticas en la enseñanza de primeras y segundas lenguas. Frente a otros métodos de análisis de la entonación, ofrece un criterio de segmentación de las melodías del habla exclusivamente fónico y presenta un sistema de procesamiento de los datos acústicos que nos permite obtener los valores relativos que constituyen las melodías, para compararlas, clasificarlas, reproducirlas con toda fidelidad, experimentar con ellas mediante la síntesis de voz, someterlas al análisis perceptivo y hacer generalizaciones lingüísticas. En Cantero \& Mateo (2011) se ofrece una visión de conjunto del método y de sus implicaciones teóricas.

El método permite contar con múltiples informantes y un gran número de contornos, que nos han servido para establecer con precisión los patrones entonativos de una lengua: del español (Cantero, 2002; Cantero et al. 2005; Cantero \& Font-Rotchés, 2007), del catalán (Font-Rotchés, 2007, 2008); así como la caracterización melódica de algunas interlenguas: el español hablado por taiwaneses (Liu, 2005), por brasileños (Fonseca, 2013), por italianos (Devís, 2011b), por húngaros (Baditzné, 2012) o por suecos (Martorell \&Font-Rotchés,2015), por citar algunos de los estudios que se han llevado a cabo.

Desde este marco teórico, se pretende dar una explicación completa de los fenómenos fónicos que tienen lugar en el habla, des de la organización jerárquica o jerarquía fónica de sus elementos (sonidos, palabras, grupos fónicos), que ejerce una función integradora y delimitadora del discurso -tratado en el concepto de entonación prelingüística-, pasando por las unidades fonológicas o tonemas, que distinguen unidades del discurso significativas -

\footnotetext{
${ }^{3}$ Para una versión en inglés Font-Rotchés y Cantero (2009).
} 
concepto de entonación lingüística-, hasta la entonación paralingüística, que da cuenta de las realizaciones expresivas, emotivas e idiosincráticas.

Para describir los modelos de entonación de les interrogativas del castellano, nos situamos en el plano de la entonación lingüística. Desde este punto de vista, en Cantero (2002) se distinguen un total de 8 tonemas en español obtenidos a partir de realizar todas las posibles

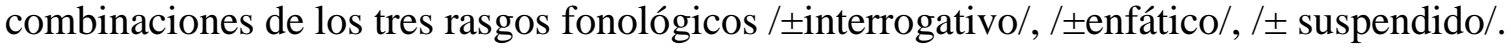
1. /+ interrog., + enfát., + susp./
5. /- interrog., + enfát., + susp./
2. /+ interrog., + enfát., - susp./
6. /- interrog., + enfát., - susp./
3. /+ interrog., - enfát., + susp./
7. /- interrog., - enfát., + susp./
4. /+ interrog., - enfát., - susp./
8. /- interrog., - enfát., - susp./

La entonación "declarativa" (o "neutra”) correspondería al tonema 8, la "interrogativa", al tonema 4, la entonación "suspendida”, al tonema 7, y la "enfática”, al tonema 6.

Cada uno de esos tonemas (cuyos rasgos melódicos están dentro de unos determinados márgenes de dispersión) puede expresarse con distintas melodías, algunas de las cuales, las más frecuentes, definimos como "melodías tipo": los patrones melódicos. Equivaldrían, pues, a las unidades descritas en el plano segmental: cada fonema puede expresarse con distintos sonidos, algunos de los cuales, los más frecuentes, definimos como "sonidos tipo". Ambos, los patrones melódicos y los sonidos tipo, son las unidades modelo de la entonación y de la pronunciación para desarrollar aplicaciones didácticas.

Para aplicar esta metodología, dividimos el proceso en dos fases, para las que empleamos el instrumento de análisis Praat (Boersma \& Weenink, 1992-2016):

- Fase acústica. En esta fase, se identifican y segmentan las unidades melódicas de los enunciados y se obtiene el valor de la frecuencia fundamental $\left(\mathrm{F}_{0}\right)$ de cada segmento tonal (en Hercios o Hz), a partir del cálculo de la mediana de los diversos valores de cada vocal que nos ofrece Praat ${ }^{4}$. Estos valores se relativizan, calculando la distancia tonal entre un segmento y el siguiente en porcentaje (que será positivo, si se trata de un ascenso, y negativo, si hay descenso), con el fin de obtener una curva estándar de la melodía. Con ello conseguimos extraer los valores relevantes de la melodía para que sean comparables independientemente de las características de sexo y edad de los informantes.

- Fase perceptiva. Los rasgos melódicos que aparecen en la curva estándar se clasifican, se comparan y se generalizan en base al análisis de las tres partes en que se pueden dividir los contornos (véanse en el gráfico 1): anacrusis, que está constituida por los segmentos tonales existentes hasta la primera vocal tónica del contorno o primer pico; cuerpo, que empieza después del primer pico y llega hasta la última vocal tónica del contorno o núcleo; e inflexión final, que es la parte más significativa del contorno y va desde la última vocal tónica hasta el final.

\footnotetext{
${ }^{4}$ Hemos publicado un script de PRAAT que, conjuntamente con una macro de Excel, permite automatizar la fase acústica del proceso (Mateo, 2010).
} 


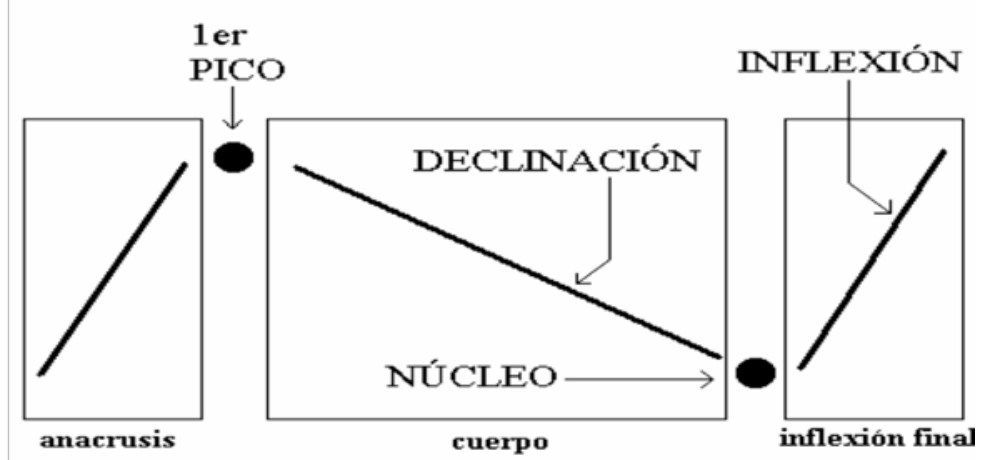

Gráfico 1. Esquema de las tres partes del contorno en Cantero \& Font-Rotchés (2007: 70).

A partir de la dirección de la inflexión final (ascendente, descendente, ascendentedescendente, descendente-ascendente, etc.) y el porcentaje del movimiento tonal, se procede a la validación perceptiva de los rasgos melódicos y de los márgenes de dispersión para establecer los patrones y su significado fonológico: / \pm interrogativo/, / \pm enfático/, $/ \pm$ suspendido/.

\section{LAS PRUEBAS PERCEPTIVAS}

$\mathrm{Al}$ analizar el corpus, constituido por 282 interrogativas, se puso de manifiesto una variedad entonativa más rica que la que se había descrito hasta el momento: se intuían más rasgos melódicos y algún patrón nuevo. Por ello, se preparó una prueba perceptiva para comprobar si las distintas melodías de determinados enunciados-pregunta se percibían como /+interrogativa/.

Como dijimos anteriormente, la inflexión final de cada contorno es el elemento más importante y, por ello, los enunciados seleccionados debían presentar diferentes porcentajes de ascenso para poder establecer, si era el caso, a partir de qué umbral, de qué porcentaje de ascenso los oyentes interpretaban que un enunciado, aislado de contexto, tiene significación interrogativa y, a su vez, cada enunciado no debía presentar ninguna marca gramatical que permitiera a los informantes de la prueba de percepción saber de qué tipo se trataba. Por estos motivos se seleccionaron 70 de los enunciados del corpus, representativos de los distintos tipos de melodías que se obtuvieron en el análisis.

Al igual que en la fase de análisis acústico, se utilizó Praat y sus opciones de síntesis y resíntesis (PSOLA) para obtener los enunciados con las inflexiones finales (variación en \%) que no habían sido producidos espontáneamente por los informantes del corpus o para crear la inflexión pertinente en los casos en que se quitó una palabra que marcaba el carácter interrogativo del enunciado.

Se dividió el corpus en dos pruebas perceptivas diferentes con 35 enunciados cada una, enunciados de los diversos patrones, tanto interrogativos como no interrogativos, y con diversas inflexiones, presentados de forma aleatoria. El informante escuchaba el archivo de audio de cada enunciado tres veces y tenía que contestar si era una pregunta, si creía que no lo era, si no lo sabía o, bien, no respondía si no era capaz de entender el significado del enunciado. El test se pasó a diferentes grupos de estudiantes de la Facultat de Formació del Professorat de la Universitat de Barcelona. De las más de 50 respuestas para cada una de las pruebas, hemos considerado únicamente las de aquellos estudiantes cuya lengua materna y 
habitual es el castellano: 32 informantes en el primer test y 33 en el segundo, muestra suficientemente representativa.

Como resultado de los tests perceptivos, se confirmó la existencia del patrón II. IF ascendente (+70\%) - descrito en Cantero et al. (2002) - con los mismos rasgos melódicos. Incluimos siete enunciados con una inflexión final del 70\% hasta el 106\% (un 100\% equivale a una octava de la escala musical), los cuales obtuvieron un 100\% de respuestas positivas, excepto dos, que fueron identificados como /+interrogativo/ por un $81 \%$ y un $84 \%$ de los informantes. En ambos casos, un 13\% de los oyentes no supo determinarlo o no contestó.

También se confirmó el patrón con inflexión final ascendente III — descrito en Cantero et al. (2002) - , pero con una variación melódica en los márgenes de la inflexión final: el ascenso que empezaba en un $40 \%$ y alcanzaba un $60 \%$, ahora se amplía hasta un $70 \%$. En las pruebas, incluimos doce enunciados con una inflexión final del 18\% hasta el 67\%. La gran mayoría de respuestas positivas, entre el $94 \%$ y el $100 \%$, se encontró en los que estaban dentro de la horquilla $40 \% \sim 70 \%$, excepto dos, que fueron identificados por un $81 \%$ de los informantes. Estos contornos tienen en común un rasgo melódico muy relevante: un primer pico desplazado en una átona posterior. Los enunciados que presentan ascensos inferiores a un $40 \%$ en la inflexión final, no presentan este primer pico desplazado o carecen de primer pico y/o cuerpo no se reconocen mayoritariamente como /+interrogativos/.

Con respecto al patrón enfático IV, con inflexión final circunfleja ascendente-descendente — descrito en Cantero et al. (2002) —, fue validado, pero con ajustes en los márgenes de la inflexión final. En las pruebas de percepción, incluimos seis enunciados con un ascenso en la primera parte de la inflexión final del 27\% hasta el 55\%. La respuestas positivas, entre un 82 $\%$ y un $100 \%$, se produjeron en los casos en los que la primera parte de la inflexión final se acerca a un $30 \%$ de ascenso o lo supera. Después, la línea melódica continúa con un descenso, que suele acabar en un punto inferior al que empezó la inflexión final y el contorno. Otro aspecto es la diferencia que se constataba entre un patrón 4a - primer pico desplazado y cuerpo con un leve descenso - y $4 \mathrm{~b}$ — sin primer pico y cuerpo plano—, a la que se nos ha añadido la presencia de contornos con un cuerpo ascendente. En el corpus actual, más amplio, los contornos suelen presentar un primer pico desplazado a una átona posterior y un cuerpo casi plano o con un descenso leve, es decir una melodía con rasgos de los patrones 4a y 4b. Por consiguiente, ante la constatación que la dirección del cuerpo no es relevante al considerar este patrón /+interrogativo/, lo caracterizaremos a partir de estos rasgos melódicos más frecuentes.

Finalmente, los contornos con unos rasgos melódicos caracterizados por un cuerpo y una inflexión final ascendentes, en las pruebas de percepción, se constató que constituyen un patrón /+interrogativo + enfático/, que nombramos patrón XIII (Font-Rotchés\&Mateo, 2011). Incluimos diecisiete enunciados con una inflexión final del $2 \%$ hasta el $66 \%$ y un ascenso total del contorno que va de $20 \%$ a $140 \%$. En este caso, observamos que los factores relevantes son: un ascenso en la inflexión final igual o superior a un $15 \%$ y un ascenso total del contorno igual o superior a un $60 \%$. Cuando los enunciados contienen ambos rasgos melódicos, un porcentaje igual o superior a un $84 \%$ de los informantes los perciben como preguntas. Cabe decir que a medida que el ascenso total del contorno es más elevado se percibe más el énfasis y, en especial, a partir de un 90\%. Existe algún enunciado que fue 
identificado como /+interrogativo/ por solo un 79\% de los informantes: se trata de ¿Entramos?, que puede ser interpretado como una invitación.

Como resultado de los tests perceptivos, constatamos que existen melodías que se perciben como interrogativas (las que responden a los rasgos de los patrones II, III, IV y XIII), las cuales vamos a tratar en los siguientes apartados. Sin embargo, hay otras que no se perciben como tales, como el patrón I con inflexión final descendente o ascendente hasta un 15\%; el patrón VI con inflexión final ascendente $(+15 \sim 70 \%)$ y primer pico en la primera tónica o inflexión final ascendente (+15 40\%) y primer pico desplazado; el patrón VII con inflexión final con núcleo elevado; y el patrón X con inflexión final descendente-ascendente. Los dos últimos, el VII y el X, deberán ser puestos a prueba de percepción con más enunciados y con más variedad de rasgos melódicos en la inflexión final. ${ }^{5}$

Al clasificar los contornos de los 282 enunciados de nuestro corpus, observamos que los informantes en habla espontánea utilizan no solo estos cuatro patrones /+interrogativos/ sino también otras melodías más propias de otro tipo de enunciados -declarativos, enfáticos o suspensos- para emitir preguntas absolutas (ver tabla 1). Así, pues, frente al 52,8\% de los contornos con melodía interrogativa, existe un $47,2 \%$, que, en principio, caracterizamos como no interrogativos. Cabe tener en cuenta que los contornos de los patrones VII y X, que constituyen un $23,4 \%$ en este grupo, deben someterse, como ya hemos comentado, a pruebas de percepción para obtener resultados concluyentes sobre sus rasgos fonológicos.

\begin{tabular}{|c|c|c|}
\hline Patrones & Núm. enunciados & Porcentaje \\
\hline /+interrogativos/ & $\mathbf{1 4 9}$ & $\mathbf{5 2 , 8 \%}$ \\
\hline II & 48 & $17 \%$ \\
\hline III & 39 & $13,8 \%$ \\
\hline IV & 30 & $10,6 \%$ \\
\hline XIII & 32 & $11,3 \%$ \\
\hline & & \\
\hline /-interrogativos/ & $\mathbf{1 3 3}$ & $\mathbf{4 7 , 2} \%$ \\
\hline I & 42 & $14,9 \%$ \\
\hline VI & 25 & $8,9 \%$ \\
\hline VII & 55 & $19,5 \%$ \\
\hline $\mathbf{X}$ & 11 & $3,9 \%$ \\
\hline Total & $\mathbf{2 8 2}$ & $\mathbf{1 0 0 \%}$ \\
\hline
\end{tabular}

Tabla 1. Los enunciados clasificados por tipo de patrón.

\section{MODELOS DE ENTONACIÓN DE LAS INTERROGATIVAS}

En este apartado, vamos a describir los cuatro patrones o melodías con valor /+interrogativo/ que hemos obtenido mediante pruebas perceptivas realizadas a hablantes nativos de español. Esto significa que un enunciado como Te cabe todo ahí o Eso lo afirma usted, sin marcas gramaticales y descontextualizado, emitido utilizando uno de estos patrones, se percibirá como una pregunta.

\footnotetext{
${ }^{5}$ Para la caracterización de estos patrones /-interrogativos/, véase Cantero y Font-Rotchés, 2007.
} 
Como ya hemos comentado al describir la constitución del corpus, estos modelos son utilizados para formular preguntas absolutas, es decir, de respuesta sí/no, preguntas retóricas (no se espera respuesta) y también enunciados interrogativos cuya fuerza ilocutiva implica otras acciones intencionales (una petición, una confirmación, un reto, una sugerencia, un ofrecimiento, entre otros). Otro tipo bien distinto serían las preguntas parciales, precedidas por un pronombre interrogativo, las cuales no necesitan una entonación de pregunta para ser reconocidas. El simple hecho de empezar el enunciado con un elemento sintáctico del tipo $a$ dónde, por qué, quien o cuando implica que el receptor capte al instante que le van a formular una pregunta.

Por consiguiente, para garantizar la percepción de una pregunta, debemos utilizar uno de estos cuatro modelos. Aun así, y como ya hemos constatado en la tabla 1, los hablantes nativos con frecuencia no los utilizan cuando se encuentran en un contexto que favorece que se perciban como una pregunta. Pero, para ello, se debe tener una buena competencia comunicativa.

A continuación, pues, vamos a comentar los rasgos melódicos de cada patrón, según los resultados del test perceptivo, aportaremos ejemplos extraídos del corpus y describiremos los usos más relevantes de cada uno, según la propuesta de Escandell-Vidal (1998a, 1998b, 2002).

\subsection{El patrón interrogativo neutro II. IF ascendente (+70\%)}

El patrón II. Inflexión final ascendente (+70\%) se caracteriza por un primer pico en la cima de un ascenso que puede llegar a ser de hasta un $40 \%$ y que puede tener lugar en la primera sílaba tónica o desplazado a una sílaba átona anterior o, como caso más frecuente, en una sílaba átona posterior a la primera tónica. El cuerpo presenta un descenso suave, seguido de una inflexión final ascendente igual o superior al 70\%, que supera de forma visible el primer pico (véase gráfico 2). Su ascenso se sitúa entre un 70\% y un $115 \%$ en todos los contornos que tenemos, excepto uno que llega a un 140\%, ¿más cositas? (Ss15). Este rasgo melódico en la inflexión final es el más importante en este tipo de contornos, porque permite interpretar como interrogativo un contorno constituido por una sola palabra ¿romanos? (AI-21) o por dos, ¿más cositas?, anteriormente citado.

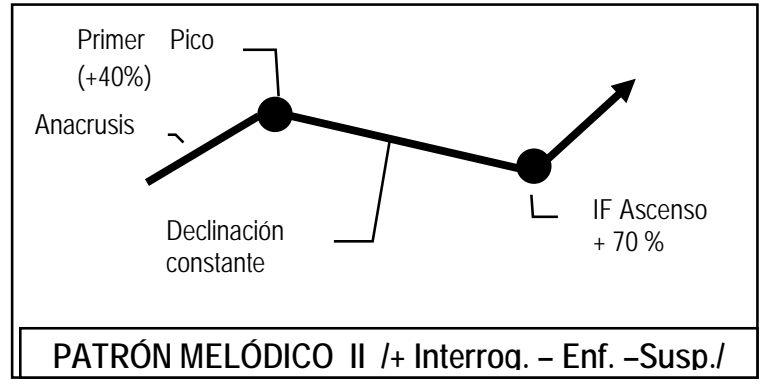

Gráfico 2. Patrón melódico II. IF ascendente (+70\%).

Así, pues, desde un punto de vista fonológico, se trata de un patrón caracterizado como /+interrogativo, -enfático, -suspenso/. Aun así, en los casos en que se superan los márgenes establecidos, es decir, si el ascenso del primer pico es superior a un $40 \%$, o en el cuerpo se da 
un descenso muy marcado o un ascenso prominente de un $140 \%$ en la inflexión final, entre otros, estaríamos ante una variante /+enfática/ de este contorno.

En nuestro corpus, hay un total de 48 contornos, un 17\%, y se utilizan básicamente en preguntas de carácter neutro en las siguientes situaciones comunicativas:

- obtener información que el emisor desconoce: ¿Alguien espera alguna llamada? (Ss1), ¿Te los has probado? (PV-01-23-02)

- hacer una pregunta de la que se conoce o se supone la respuesta: ¿Tú has perdido algún quilo? (Ss34), ¿Nos sacas una tanga para ver cómo es? (PV-01-12-01)

- confirmar algo: ¿Y de eso ha mejorado no, Antonia? (AN-63-01-07), ¿Eso lo afirma usted? (M-02-04-04), ¿Romanos? (AI-21)

- realizar una petición: ¿Me lo puedes repetir? (CM-10-05-01)

- pedir permiso: ¿Me puedo quitar la corbata también? (MU-13-04-09)

- un uso retórico Que será lo mismo, ¿no? (AI-22)

Los enunciados, como podemos ver en los gráficos 3 y 4, responden todos al contorno melódico del patrón II: primer pico desplazado a una vocal átona posterior, cuerpo descendente e inflexión final igual o superior a un $+70 \%$.

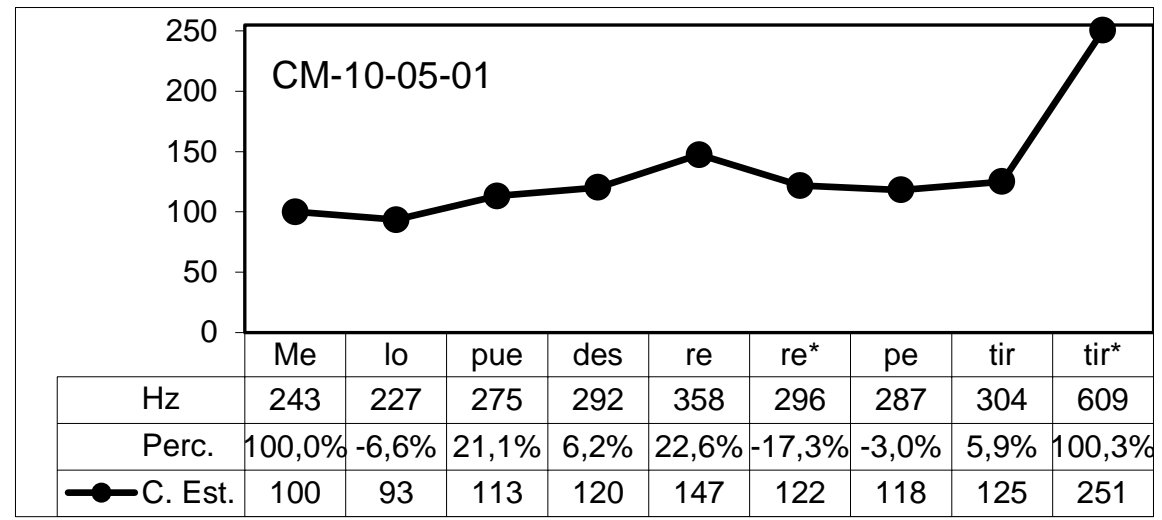

Gráfico 3. Contorno típico del patrón melódico II: ¿Me lo puedes repetir?

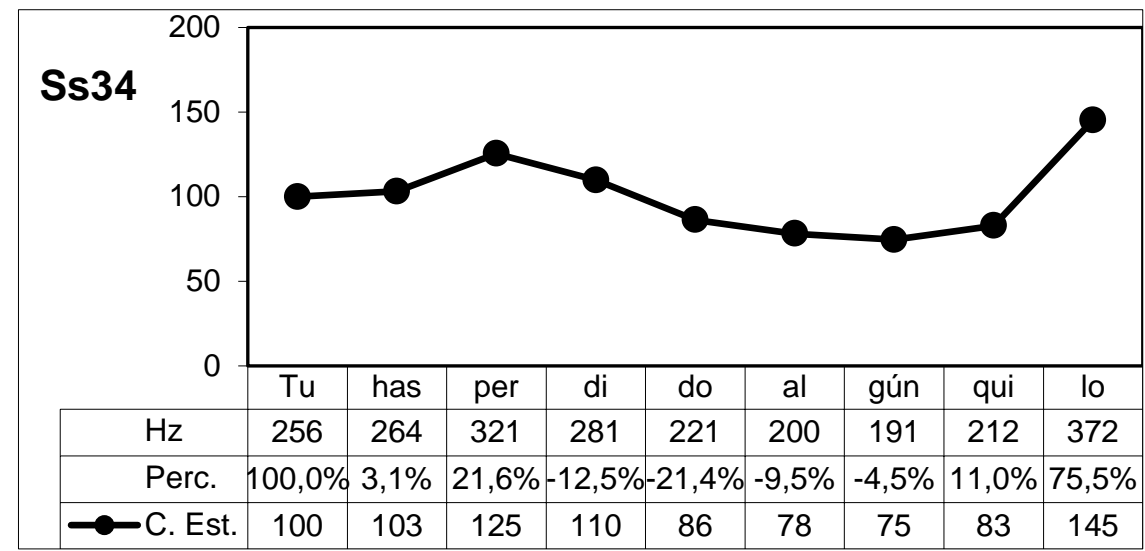

Gráfico 4. Contorno típico del patrón melódico II: ¿'Tú has perdido algún quilo? 


\subsection{El patrón interrogativo neutro III. IF ascendente (40\% 70\%)}

El patrón III. Inflexión final ascendente (+40\% 70\%) se caracteriza por presentar un primer pico en la cima de un ascenso desplazado a una sílaba átona posterior a la primera tónica, que puede llegar a ser considerable (un 60\%). El cuerpo presenta un descenso suave, seguido de una inflexión final ascendente ( $+40 \% \sim 70 \%)$, que también en este caso suele superar de forma visible el primer pico (véase gráfico 5).

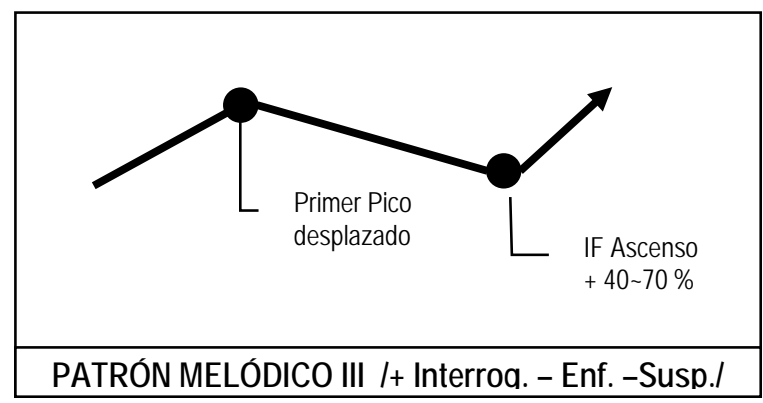

Gráfico 5. Contorno típico del patrón melódico III.

A diferencia del patrón II, el rasgo melódico del primer pico junto con una inflexión final que supere el $40 \%$ son las dos características que debe cumplir un contorno para ser reconocido como pregunta en castellano. En realidad, son patrones complementarios, con la diferencia que los contornos del patrón II se reconocen solo con una inflexión final, mientras que los del III, se deben presentar completos y con un primer pico desplazado. Así, pues, desde un punto de vista fonológico, se trata de un patrón caracterizado con el mismo tonema que el patrón II: /+interrogativo, -enfático, -suspenso/.

En nuestro corpus, hay un total de 39 contornos, un 13,8\%, y tienen lugar en preguntas de carácter neutro para los siguientes usos:

- obtener información que el emisor desconoce: ¿Y te fijaste si el pantalón tejano era elástico? (AI-10), ¿Se puede superar este miedo? (Ss33)

- hacer una pregunta de la que se conoce o se supone la respuesta: ¿Era imposible? (CA-39-04-03), ¿Tú tienes novia? (Ss42)

- confirmar algo: ¿Hay que esperar tres años para visitarla? (AI-30), ¿Es una indirecta para que me vaya? (MU-10-03-01)

- realizar una petición: ¿Alguien me llama por teléfono? (MU-10-04-05)

- un ofrecimiento ¿Te apetece que vayamos a verla? (CA-91-04-03)

- pedir permiso ¿Puedo contar un chiste? (CM-92-01-06).

Ambos patrones, el II y el III, tienen los mismos usos, pero cabe decir que no hemos encontrado ningún ejemplo de pregunta retórica. Aun así, es muy probable que existan.

En los siguientes gráficos, 6 y 7, vemos dos ejemplos de este patrón. El contorno del gráfico 6 es un caso típico de este patrón, ya que presenta un ascenso en el primer pico de un 37\%, $Y$ te fijaste, que culmina en una sílaba átona posterior a la primera tónica -te, y, a su vez, una inflexión final de un 50\%, que empieza en -lás- y sigue hasta -co. Se trata de una pregunta para obtener información que el emisor desconoce. En cambio, con una melodía 
perteneciente al mismo patrón, el enunciado ¿Alguien me llama por teléfono? tiene lugar en un contexto en el que una concursante hace la petición de que le llamen.

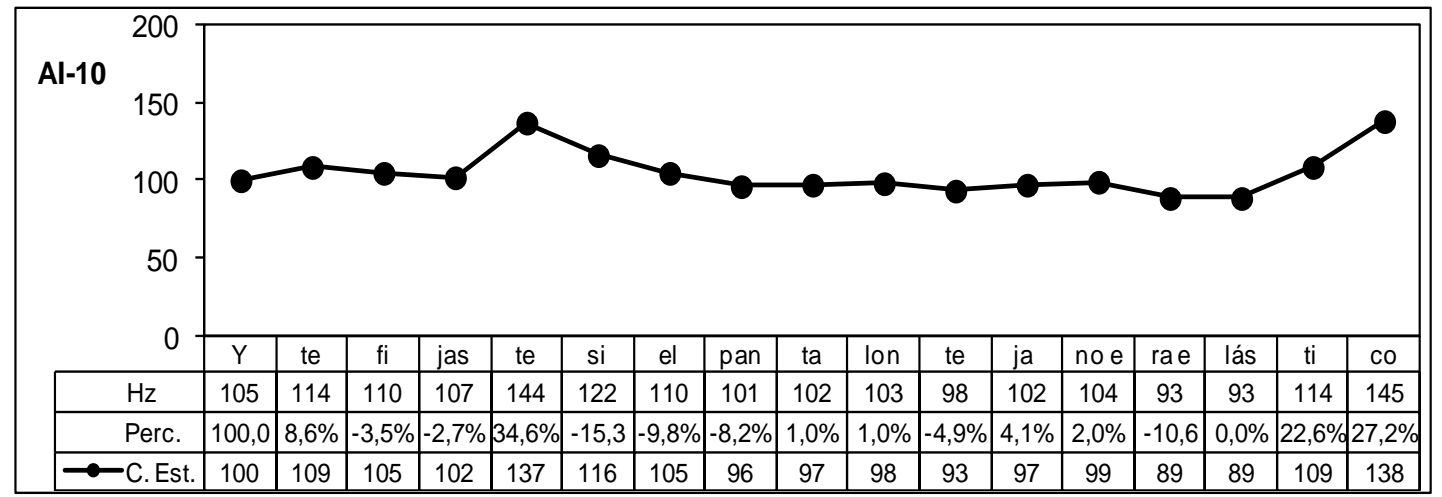

Gráfico 6. Contorno típico del patrón melódico III: ¿Y te fijaste si el pantalón tejano era elástico?

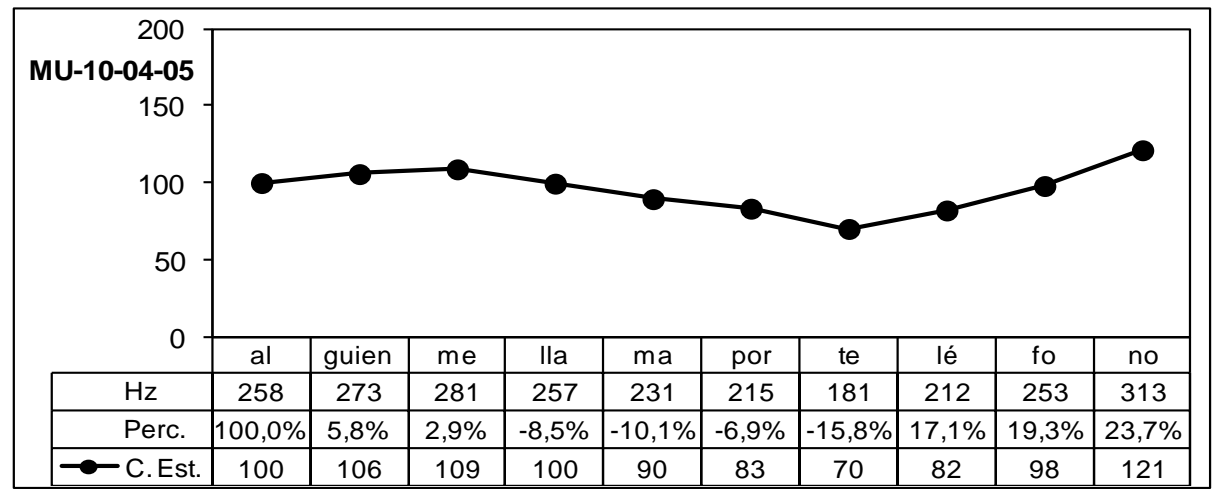

Gráfico 7. Contorno típico del patrón melódico III: ¿Alguien me llama por teléfono?

El contorno del gráfico 7 presenta un primer pico desplazado a una átona posterior, Alguien me, pero con un ascenso poco marcado de un 9\%, ante una inflexión final de un 43\%, que va de -lé - a -no, característica de este patrón.

\subsection{El patrón interrogativo enfático. IV. Inflexión final ascendente-descendente}

El patrón IV. Inflexión final ascendente-descendente (véase gráfico 8). Este patrón presenta un primer pico desplazado a una sílaba átona posterior, un cuerpo plano o con un descenso suave y una inflexión final ascendente-descendente, en la que el ascenso tiene que ser igual o superior a un 30\%. ${ }^{6}$ Aunque definimos el cuerpo a partir de los rasgos melódicos de la mayoría de contornos obtenidos, tenemos algunos casos en los que el cuerpo es ascendente.

\footnotetext{
${ }^{6}$ Referente a este patrón, hemos observado que algunos autores (Estevas-Vilaplana y Prieto, 2010, Vizcaíno y Cabrera, 2011, entre otros) hablan de inflexión circunfleja y todo parece indicar que no hacen la distinción entre este patrón IV y el patrón VII propuestos por AMH. Desde este método, para que una inflexión final sea circunfleja el primer valor debe empezar en la última sílaba tónica y presentar un total de tres valores y dos direcciones. La melodía del patrón VII. IF de núcleo elevado se caracteriza por una inflexión final que empieza en la última sílaba tónica, que está situada en una cima y va seguida por un descenso con un total de dos valores y una dirección, un descenso. Aparentemente, se parecen mucho, pero no son iguales.
} 


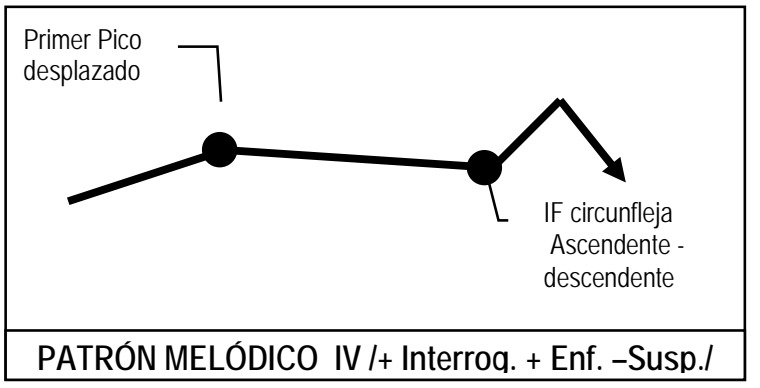

Gráfico 8. Contorno típico del patrón melódico IV.

Así, pues, desde un punto de vista fonológico, se trata de un patrón caracterizado como /+interrogativo, +enfático, -suspenso/.

En nuestro corpus, tenemos un total de 30 contornos, un 10,6\%, los cuales tienen usos muy parecidos a los patrones neutros - excepto peticiones, ofrecimientos y usos retóricos-, pero con un añadido de cortesía. El alargamiento de una de las vocales de la inflexión final es un elemento atenuador que suaviza la pregunta, como ya constató Devís (2011a) en pruebas de percepción:

- obtener información que el emisor desconoce: ¿Te gusta el tomate? (MU-01-11-06), ¿Y quién viene, Juani? (M-06-02-05), ¿Les gustan las castañas? (AN-26-01-08)

- hacer una pregunta de la que conoce o supone la respuesta: ¿Y a las fiestas de Barranco no habéis ido nunca? (EX 01-01-03)

- confirmar algo: ¿Lo dices en serio? (Ss27), ¿Seguro? (Ss43), ¿La trashumancia? (AR04-01-03)

- pedir permiso ¿Se puede?( CM-93-03-01)

En el gráfico 9, la pregunta ¿Les gustan las castañas? tiene la finalidad de obtener información que el emisor desconoce. La melodía del contorno empieza con un ascenso muy marcado de un 63\% hasta el primer pico, -tan, seguido de un cuerpo que va descendiendo, con algunas resituaciones, hasta la última sílaba tónica, -ta. Continúa con el ascenso de la inflexión final, de un 30,2\%, y un descenso, algo truncado, ya que no culmina en un punto inferior al del inicio de la inflexión. 


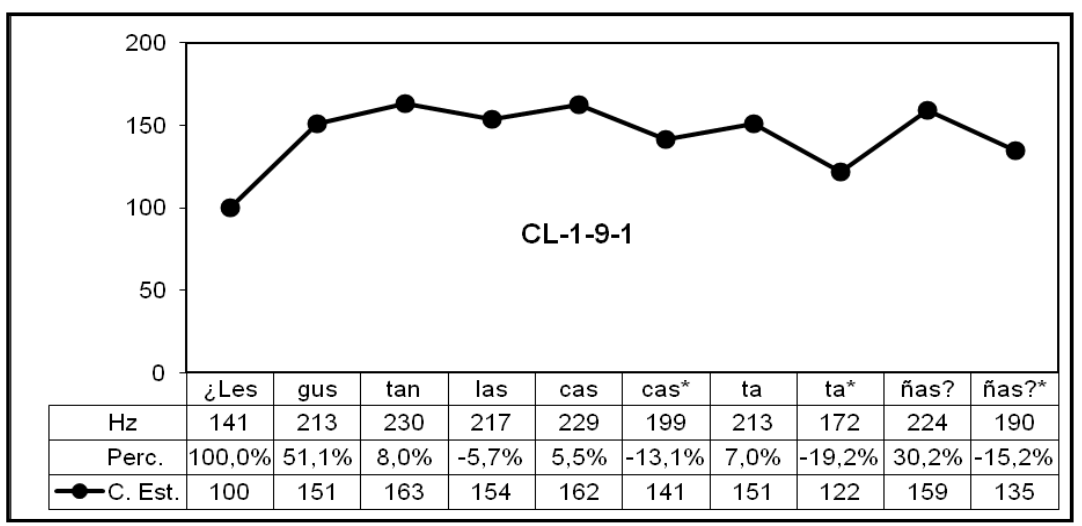

Gráfico 9. Contorno típico del patrón melódico IV: ¿Les gustan las castañas?

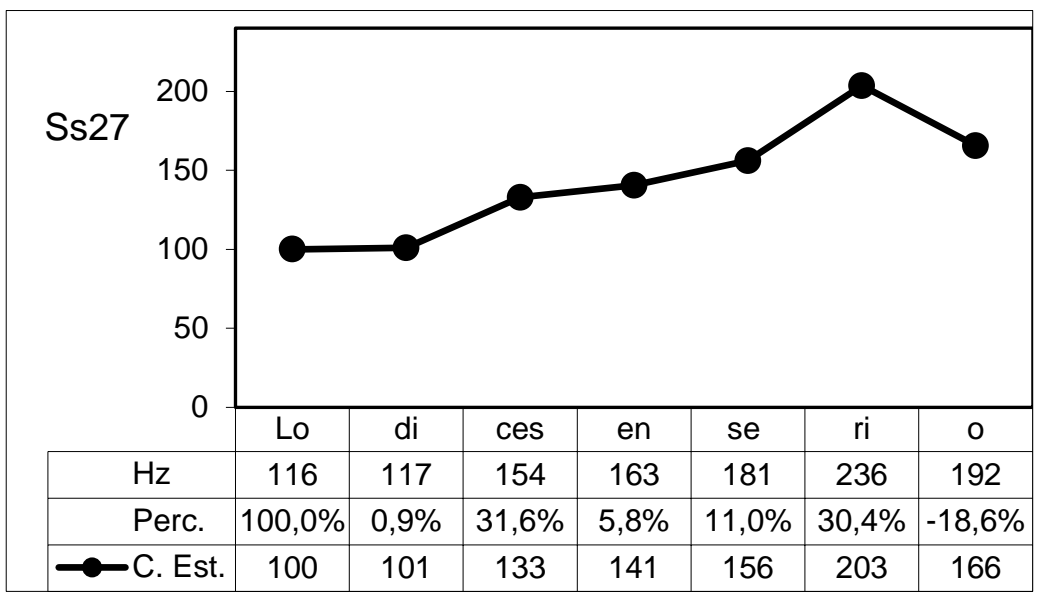

Gráfico 10. Contorno típico del patrón melódico IV: ¿Lo dices en serio?

En el gráfico 10, encontramos una pregunta confirmatoria ¿Lo dices en serio?. Su melodía se caracteriza por un cuerpo ascendente - ya habíamos comentado que había algunos enunciados con esta tendencia, aunque no es la más frecuente- - y sin primer pico, que sigue hasta la sílaba tónica se-. A partir de esta sílaba, que se encuentra en un punto elevado, tiene lugar un ascenso hasta $-r i$, de un 30,4\%, y a continuación un descenso hasta - - , de un $18,6 \%$, que no alcanza el punto donde había empezado la inflexión final.

\subsection{El patrón interrogativo enfático XIII. Cuerpo e inflexión final ascendentes}

El patrón XIII. Cuerpo e inflexión final ascendentes (véase gráfico 11). Se caracteriza por un cuerpo ascendente, frecuentemente sin primer pico, y una inflexión final ascendente. En este caso, la inflexión final suele ser de un $15 \%$ o superior, aunque el rasgo melódico que parece determinante es el ascenso total que tiene lugar en el contorno, que tiene que ser igual o superior a un $60 \%$. Cabe decir que cuanto más elevado es el ascenso - a partir de un $90 \%$ de ascenso total—, más se percibe el énfasis. 


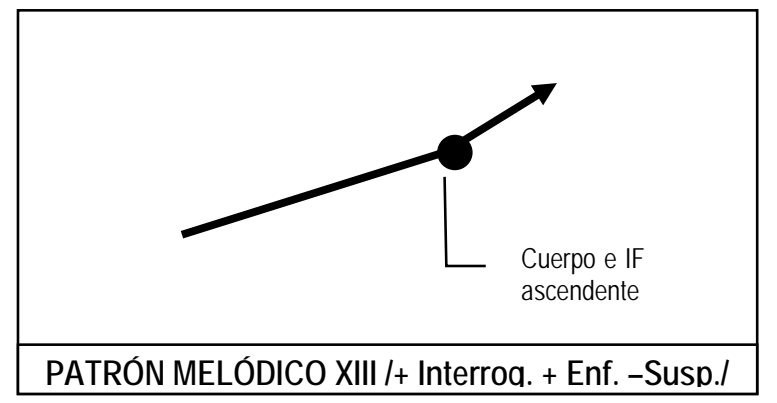

Gráfico 11. Contorno típico del patrón melódico XIII.

Así, pues, desde un punto de vista fonológico, se trata de un patrón caracterizado como /+interrogativo, +enfático, -suspenso/. En nuestro corpus, tenemos un total de 32 contornos, un $11,3 \%$. Se utilizan básicamente en preguntas exclamativas en las que "el emisor está favoreciendo también la cortesía positiva porque demuestra un interés evidente por todo lo relacionado con el destinatario" (Escandell-Vidal, 1998:181), en casos como, ¿Me oyes? (Ss38), ¿Me sigues? (AN-82-06-01), ¿Un poquito de laca te ponemos? (M-06-02-01), ¿Y te gusta viajar? (AN-26-02-09). Este tipo de melodía con un matiz de cortesía puede comportar otros significados pragmáticos adicionales:

- Una sugerencia: ¿Me pones un quilo de plátanos? (AR-05-11-03), ¿Le quieres dar un chicle al cámara? (AR-05-14-06)

- Pedir permiso: ¿Me puedo levantar y cantar ya? (AN-25-05-03)

- Un ofrecimiento: ¿Queréis ver el jardín? (M-08-03-01)

- Sorpresa ¿De Barcelona también? (Ss40), ¿Pero habéis estado allí? (CM-01-02-09)

- Un reto ¿Te atreves? (EX-46-09-03)

Veamos a continuación dos contornos típicos de este patrón, gráficos 12 ¿Pero habéis estado allí?, con un significado adicional de sorpresa, y 13, ¿Queréis ver el jardín?, en el que el interés por el destinatario se ve complementado por un ofrecimiento o invitación.

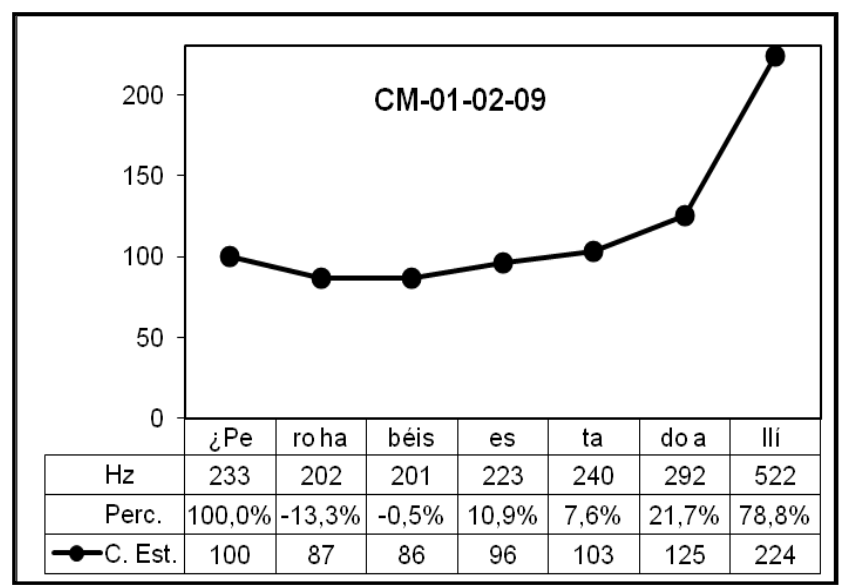

Gráfico 12. Contorno típico del patrón melódico XIII: ¿Pero habéis estado allí?.

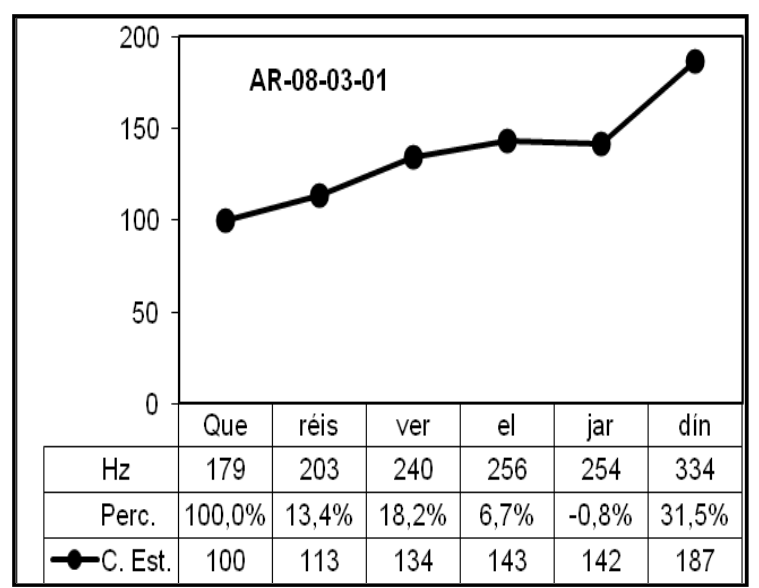

Gráfico 13. Contorno típico del patrón melódico XIII: ¿Queréis ver el jardín?

Ambos contornos, gráficos 12 y 13, presentan un cuerpo ascendente y una inflexión final también ascendente y suman un 160\% y un $187 \%$ de ascenso, respectivamente, con lo cual se caracterizan, según las pruebas de percepción, por ser enfáticos. 


\section{CONCLUSIONES}

Aunque toda una larga tradición de trabajos sobre entonación se ha preocupado por describir las interrogativas absolutas, los manuales de aprendizaje de español para extranjeros todavía no tratan este aspecto de la comunicación oral tan importante para evitar ambigüedades y malentendidos y favorecer la comunicación. En cambio, la Nueva gramática de la lengua española. Fonética y fonología (RAE, 2011), que sí que contiene un capítulo de entonación con un apartado dedicado a las interrogativas absolutas o totales, no contempla toda la riqueza entonativa que conocemos hasta nuestros días, en cuanto a patrones distintos, a sus características melódicas y los diversos usos pragmáticos en distintos contextos.

Ante la evidencia de la existencia de esta riqueza entonativa, ya apuntada hace años por miembros del Laboratorio de Fonética Aplicada de la UB, hemos llevado a cabo una investigación más amplia a partir de un corpus de 282 interrogativas, emitidas por 197 informantes, y de unas pruebas de percepción. La finalidad era profundizar en el establecimiento y descripción de los patrones interrogativos, sus rasgos melódicos y sus usos pragmáticos para aplicarlos a la enseñanza del español.

Hemos establecido cuatro patrones o modelos entonativos, dos neutros y dos enfáticos. Los patrones neutros II y III, con inflexión final ascendente, son complementarios. El patrón II presenta un ascenso en la inflexión final igual o superior a un 70\%, mientras que, en el III, este ascenso se sitúa entre un $40 \%$ y un $70 \%$, rasgo que se complementa con un primer pico desplazado a una átona posterior, que es imprescindible. Estos patrones se utilizan en preguntas para obtener información que el emisor desconoce o, bien, en preguntas en las que supone la respuesta, o que son para confirmar algo, para realizar una petición o un ofrecimiento, para pedir permiso o para formular una pregunta retórica.

El patrón enfático IV con IF ascendente-descendente se caracteriza por presentar un primer pico desplazado a una átona posterior, un cuerpo casi plano o con un ligero descenso, y el ascenso de la inflexión final debe alcanzar un 30\% seguido de un descenso. Puede darse el caso de que algunos contornos presenten un cuerpo plano o ascendente sin primer pico, pero no supone ningún problema comunicativo, ya que la información entonativa de pregunta está contenida en la inflexión final. Sus usos, obtener información más o menos conocida o confirmar, que coinciden con los patrones neutros, aportan un rasgo atenuador de cortesía, que les distingue.

Finalmente, el patrón XIII, de cuerpo e inflexión final ascendentes, se caracteriza por una inflexión final igual o superior a un $15 \%$ y un ascenso total del contorno del $60 \%$ o superior. Es propio de las preguntas exclamativas, pero también puede aportar otros significados pragmáticos, como sugerencias, retos, ofrecimientos o pedir permiso.

Ahondar en las interrogativas absolutas nos ha permitido confirmar el patrón XIII, establecer variaciones en los rasgos melódicos de los patrones III y IV ya descritos, y profundizar en los usos pragmáticos.

Gracias a las pruebas de percepción, también hemos constatado que aparte de estas cuatro melodías, reconocidas como /+interrogativas/ existen otras que, aunque descontextualizadas no se perciben como preguntas, pueden usarse como preguntas en el contexto. Se trata de los contornos que siguen el patrón I con inflexión final descendente, propio de las declarativas, y el patrón VI, propio de los enunciados suspensos, con inflexión final ascendente hasta un 
40\%, o hasta un 70\%, con la restricción que el primer pico se encuentre en la primera sílaba tónica.

A su vez, nos ha planteado más cuestiones y nuevos retos. En este sentido, en un futuro debemos realizar pruebas de percepción para los contornos de los patrones VII, IF con núcleo elevado, que es el que tiene más presencia en nuestro corpus, con un 19,5\% de los contornos, aunque cabe decir que en gran parte de los ejemplos la elevación del núcleo es poco marcada (inferior a un 25\%) y X, IF descendente-ascendente, que es el que tiene menor presencia, un $3,9 \%$, así como también estudiar sus usos pragmáticos a partir de corpus audiovisuales que sean un reflejo de la lengua que utilizan los hablantes de español.

\section{REFERENCIAS BIBLIOGRÁFICAS}

Álvarez Martínez, M. Á.; Canales, A. B.; Torrens Álvarez, M. J. y Alarcón Pérez, C. (2005): Vuela 1, Universidad de Alcalá, Editorial Anaya.

Baditzné Pálvölgyi, Kata (2012): Spanish Intonation for Hungarian learners: yes/no questions. Biblioteca Phonica, 15. http://revistes.ub.edu/index.php/phonica/article/view/ 11041. (Consultado el 25/05/17).

Baditzné Pálvölgyi, Kata y FONT-ROTCHÉS, Dolors (2013): “The intonation of Spanish yes/no questions in spontaneous speech and a didactic material”. Beszédkutatás 2013, 76-93.

Ballesteros, Mapi (2011): La entonación del español del norte. Tesis doctoral. Dep. de Filología Hispánica, Universitat de Barcelona. http://diposit.ub.edu/dspace/bitstream/ 2445/35060/3/02.MBP_ANEXOS.pdf. (Consultado 23/05/17).

Ballesteros, Mapi; Miguel Mateo y Francisco José Cantero Serena (2010): “Corpus oral para el análisis melódico de las variedades del español”. Actas del XXXIX Simposio Internacional de la SEL. Santiago de Compostela: Univ. de Santiago de Compostela.

Boersma, Paul; Weenink, David (1992-2016): PRAAT. Doing phonetics by computer. Institute of Phonetic Sciences, University of Amsterdam. (Disponible en http://www.praat.org).

Cantero Serena, Francisco José (2002): Teoría y análisis de la entonación. Barcelona: Edicions de la Universitat de Barcelona.

Cantero Serena, Francisco José; M. A. de Araújo; Y.H. Liu; Y.K. Wu y A. Zanatta (2002): "Patrones melódicos de la entonación interrogativa del español en habla espontánea". Actas del II Congreso de Fonética Experimenta. Sevilla: Univ. Sevilla, 118-123.

Cantero Serena, Francisco José; Raúl Alfonso; Marta Bartolí; Anna Corrales y Maribel Vidal (2005): “Rasgos melódicos de énfasis en español”, Phonica, 1.

Cantero Serena, Francisco José y Dolors Font-Rotchés (2007): "Entonación del español peninsular en habla espontánea: patrones melódicos y márgenes de dispersión”. Moenia, 13, 69-92.

Cantero Serena, Francisco José y Dolors Font-Rotchés (2009): "Protocolo para el análisis melódico del habla”. Estudios de Fonética Experimental, XVIII, 17-32.

Cantero Serena, Francisco José y Miguel Mateo-Ruiz (2011): “Análisis Melódico del Habla: complejidad y entonación en el discurso”. Oralia, 14, 105-127. 
Chamorro, María Dolores et al. (2006): El ventilador. Barcelona: Difusión.

Congosto, Yolanda (2011): “Continuum entonativo: declarativas e interrogativas absolutas en cuatro variedades del español peninsular y americano”. Revista Internacional de Lingüística Iberoamericana, IX, 75-90.

Devís Herraiz, Empar (2011a): “Entonación de (des)cortesía en el español coloquial”, Phonica, 7, 36-79.

Devís Herraiz, Empar (2011b): “La entonación del español hablado por italianos”. Didáctica (Lengua y Literatura), 23, 35-58.

Dorta, Josefa y Beatriz Hernández (2005): “Análisis prosódico de un corpus de habla experimental: interrogativas absolutas con expansión en el objeto vs sin expansión”. Estudios de Fonética Experimental, XIV, 67-123.

Dorta, Josefa. (ed.) (2007): La prosodia en el ámbito lingüístico románico. Santa Cruz de Tenerife: La Página Ediciones.

Escandell-Vidal, M. Victoria (1998a): "Intonation and Procedural Encoding: The Case of Spanish Interrogatives”. En V. Rouchota y A. Jucker (eds.): Current Issues in Relevance Theory. Amsterdam: John Benjamins, 169-203.

Escandell-Vidal, M. Victoria (1998b): "Los enunciados interrogativos. Aspectos semánticos y pragmáticos”. En I. Bosque y V. Demonte (eds.): Nueva Gramática Descriptiva de la Lengua Española. Madrid: Instituto universitario Ortega y Gasset, 3929-3992.

Escandell-Vidal, M. Victoria (2002): Introducción a la Pragmática. Barcelona: Ariel.

Elejabeitia, Ana; Alexander Iríbar y Rosa Pagola (2005): "Notas sobre la prosodia del castellano en Bizkaia”. Estudios de Fonética Experimental, XIV, 247-272.

Estebas-Vilaplana, Eva y Pilar Prieto (2010): “Castilian Spanish intonation”. En Prieto, Pilar y Paolo Roseano (coords.): Transcription of Intonation of the Spanish Language. München: Lincom Europa, 17-48.

Face, Timothy L. (2004): “The intonation of absolute interrogatives in Castilian Spanish”. Southwest Journal of Linguistics, 23 (2), 65-80.

Fonseca, Aline (2013): Caracterización de la entonación del español hablado por brasileños. Tesis doctoral. Dep. Didàctica de la Llengua i la Literatura. Universitat de Barcelona. http://www.tesisenred.net/handle/10803/134929. (Consultado 23/05/17).

Font-Rotchés, Dolors (2007): L'entonació del català. Barcelona: Publicacions de l’Abadia de Montserrat.

Font-Rotchés, Dolors (2008): “Els patrons entonatius de les interrogatives absolutes del català central”. Llengua i Literatura, 19, 299-329.

Font-Rotchés, Dolors y Francisco José Cantero Serena (2009): "Melodic Analysis of Speech Method applied to Spanish and Catalan”. Phonica, 5, 33-47. http://www.publicacions.ub.edu/revistes/phonica5/Default.asp.

Font-Rotchés, Dolors y Mateo-Ruiz, Miguel (2011): “Absolute interrogatives in Spanish, a new melodic pattern”. Actas del VII Congresso Internacional da ABRALIN. Curitiba: 1111-1125. 
Font-Rotchés, Dolors y Mateo-Ruiz, Miguel (2013): "Entonación de las interrogativas absolutas del español peninsular del sur en habla espontánea”. Onomázein, 28, 256-275.

Liu, Yen-Hui (2005): La entonación del español hablado por taiwaneses, Biblioteca Phonica, 2. http://www.publicacions.ub.es/revistes/phonica-biblioteca/esp_taiw/esp_ taiw.pdf. (Consultado 23/05/17).

Martínez Celdrán, Eugenio (2011): "La línea melódica de la entonación declarativa e interrogativa absoluta en el español de España”. En Hidalgo, Antonio; Yolanda Congosto y Mercedes Quilis (eds.): El estudio de la prosodia en España en el siglo XXI: perspectivas y ámbitos. Valencia: Universitat de València, Anejo 75 de Quaderns de Filologia.

Martínez Celdrán, Eugenio y Fernández, Anna M. (2003): “Taxonomía de las estructuras entonativas de las modalidades declarativa e interrogativa del español estándar peninsular”. En Herrera, Esther y Pedro Martín Butragueño (eds.) La tonía: dimensiones fonéticas y fonológicas. México: El Colegio de México.

Martorell, Laura; Font-Rotchés, Dolors (2015): "Es un hombre famoso o ¿Es un hombre famoso? Rasgos melódicos de las interrogativas absolutas dels español hablado por suecos. En Cabedo, Adrián (coord.): Perspectivas actuales en el análisis fónico del habla. Tradición y avances en la fonética experimental. Anejo n.7 de Normas. Revista de estudios lingüísticos hispánicos.

Mateo-Ruiz, Miguel (2010): "Protocolo para la extracción de datos tonales y curva estándar en Análisis Melódico del Habla (AMH)”. Phonica, 6, 49-90. http://www.publicacions.ub.edu/revistes/phonica6/Default.asp (Consultado 4/09/2012).

Mateo-Ruiz, Miguel (2014): La entonación del español meridional. Tesis doctoral. Dep. Didàctica de la Llengua i la Literatura. Universitat de Barcelona. http://www.tdx.cat/handle/10803/132583. (Consultado 23/05/17).

Navarro Tomás, Tomás. (1944): Manual de entonación española, New York: Hispanic Society (1974 ${ }^{4}$ ); Madrid: Guadarrama.

Quilis, Antonio (1989): "La entonación de Gran Canaria en el marco de la entonación española”. Lingüística Española Actual, XI-I, 55-88.

Ramírez, Ma Dolores (2005): “Aproximación a la prosodia de Madrid”. Estudios de Fonética Experimental, XIV, 309-326.

Real Academia Española (2011): Nueva gramática de la lengua española. Fonética y fonología. Barcelona: Espasa Libros, S.L.U, 435-488.

Sans Baulenas, Neus y Ernesto Martín Peris (2004): Gente 1 y 2. Barcelona: Difusión.

Sperber, Dan y Deirdre Wilson (1986): Relevance. Communication and Cognition. Oxford: Blackwell.

Vizcaíno, Francisco y Mercedes Cabrera (2011): "Semántica procedimental y entonación de los enunciados interrogativos de Las Palmas de Gran Canaria”. En Hidalgo, Antonio, Yolanda Congosto y Mercedes Quilis (eds.) : El estudio de la prosodia en España en el siglo XXI: perspectivas y ámbitos. Valencia: Universitat de València, Anejo 75 de Quaderns de Filologia.

Wilson, Deirdre y Dan Sperber (1993): “Linguistic Forma and Relevance”. Lingua 93, 1-25. 